\title{
A MINIMUM CONDITION AND SOME RELATED FIXED-POINT THEOREMS
}

\author{
JACEK R. JACHYMSKI and JAMES D. STEIN, JR.
}

(Received 19 May 1998; revised 17 September 1998)

Communicated by E. N. Dancer

\begin{abstract}
The classic Banach Contraction Principle assumes that the self-map is a contraction. Rather than requiring that a single operator be a contraction, we weaken this hypothesis by considering a minimum involving a set of iterates of that operator. This idea is a central motif for many of the results of this paper, in which we also study how this weakened hypothesis may be applied in Caristi's theorem, and how combinatorial arguments may be used in proving fixed-point theorems.
\end{abstract}

1991 Mathematics subject classification (Amer. Math. Soc.): primary 47H10, 54H25.

Keywords and phrases: metric space, asymptotically regular map, contraction, uniformly continuous map, semi-continuous function, fixed point, periodic point.

\section{Introduction}

Since arbitrary self-maps do not, in general, have fixed points, in order to ensure the existence of fixed points it is necessary to place additional hypotheses on the maps. A productive class of hypotheses involves specifying how the map behaves on certain pairs of points. For example, the Banach Contraction Principle requires that $d(T x, T y) \leq M d(x, y)$ for some positive constant $M<1$. Caristi's Fixed-point Theorem requires that there exists a lower semi-continuous map $\alpha: X \mapsto \mathbf{R}_{+}$such that, for each $x \in X, d(x, T x) \leq \alpha(x)-\alpha(T x)$. In the Banach Contraction Principle, the restriction is applied to all pairs $(x, y)$; in Caristi's Theorem, the restriction is applied to pairs of the form $(x, T x)$.

Suppose that $\mathscr{F}$ is a set of self-maps of a set $X$. Instead of requiring that a single operator $T$ satisfy a restriction for certain pairs belonging to $X \times X$, we might instead require that, for each pair in a given collection of pairs, there exists an operator

(C) 1999 Australian Mathematical Society 0263-6115/99 \$A2.00+0.00 
$T \in \mathscr{F}$ (dependent on the pair) satisfying that restriction. To illustrate, we consider the following conjectured generalization of the Banach Contraction Principle. Rather than requiring that a single operator be a contraction, we consider a minimum involving a set of iterates of that operator. This idea is a central motif for many of the results of this paper.

GeNeralized BanaCh ConTRACTION CONJECTURE (GBCC). Let $(X, d)$ be $a$ complete metric space, $0<M<1$, and $T$ a self-map of $X$. Let $J$ be a finite set of positive integers. Assume that $T$ satisfies the following condition:

$$
\min \left\{d\left(T^{k} x, T^{k} y\right): k \in J\right\} \leq M d(x, y) .
$$

Then $T$ has a fixed point.

This conjecture has been proved when $J=\{1\}$ (by Banach), and for the cases $J=\{1,2\}, J=\{1,3\}$, and $J=\{2,3\}$ (in Jachymski-Schröder-Stein [8]); it is then shown that if $p$ is a positive integer, these results imply GBCC for the cases $J=\{p, 2 p\}, J=\{p, 3 p\}$, and $J=\{2 p, 3 p\}$. An alternative way of phrasing the above conjecture (see Stein [9] for a more thorough discussion, where an example is also given that GBCC is, in general, false for infinite sets $\mathscr{F}$ ) is to assume that, for each $x, y \in X$, there is an operator $Q=Q_{(x, y)} \in\left\{T^{j}: j \in J\right\}$ such that $d(Q x, Q y) \leq M d(x, y)$.

An example is given in Jachymski-Schröder-Stein [8] of a discontinuous operator $T$ on a complete metric space $X$ which satisfies (1) for $J=\{1,2,3\}$. Obviously, the larger the set $J$, the less restrictive the hypothesis. Even in the case $J=\{1,2\}$, it is possible to construct continuous maps which are far from contractive; we give an example to illustrate.

The example is somewhat on the computational side, but can be described fairly easily. $X$ consists of the countable union of planar sets, each of which contains the origin as the fixed point and consists of isolated points on two parallel lines (the $x$-axis and a line far away). The distances of the points on each side of each line are geometrically increasing, but all the points on the right side of each line are extremely close to each other when compared to the points on the left side of each line.

On one line, the map $T$ essentially flips the points from one side to the other, such that $T^{2}$ brings each point closer to the origin. This has the effect of making $d\left(T^{2} x, T^{2} y\right) / d(x, y)$ small, but drastically increases $d\left(T^{2 k-1} x, T^{2 k-1} y\right)$ for $x$ and $y$ both on the closely-spaced side of the line. On the second line, the map $T$ takes the points into the first line in such a way that $d(T x, T y) / d(x, y)$ is small, but drastically increases $d\left(T^{2 k} x, T^{2 k} y\right)$ for points on the closely-spaced side of the second line.

Each of these 'two-line' sets is finite, and $T^{k}$ is eventually constant on each of them. It is therefore necessary to paste together countably many such 'two-line' sets. The basic metric used is also known as the 'river metric' in Engelking [6]. 
EXAMPLE 1. Let $0<M<1$. We construct a complete metric space $(X, d)$ and a continuous map $T: X \mapsto X$ such that:

$$
\begin{gathered}
\min \left\{d(T x, T y), d\left(T^{2} x, T^{2} y\right)\right\} \leq M d(x, y) \\
\text { no } T^{k} \text { is a contraction for any } k .
\end{gathered}
$$

$X$ will consist of a countable union of spaces $W_{n}$ whose intersection consists of a single point, which will be the fixed point of the map $T$.

We start by assuming that $a>0$ and $R>1$ are arbitrary, and that $N$ is a positive integer. We shall construct a subset $X_{N}=X_{N}(a, R)$ of the plane $\mathbf{R}^{2}$ which consists of isolated points on two parallel lines.

Assume that $r>R$ (we shall later specify how to choose $r$ ), and that $p$ is an integer, also to be specified later. Define

$$
A_{N}=A_{N}(a, r)=\{(0,0)\} \cup\left\{\left(a r^{k}, 0\right): 0 \leq k \leq N\right\} \cup\left\{\left(-a r^{k}, 0\right): N \leq k \leq 2 N\right\} ;
$$

$A_{N}$ is a subset of the $x$-axis of cardinality $2 N+3$. Let

$$
\begin{aligned}
B_{N} & =B_{N}(a, r) \\
& =\left\{\left(a r^{k}, a r^{2 N+p}\right): 1 \leq k \leq N\right\} \cup\left\{\left(-a r^{k}, a r^{2 N+p}\right): N+1 \leq k \leq 2 N\right\} ;
\end{aligned}
$$

$B_{N}$ is a subset of the line $y=a r^{2 N+p}$ consisting of $2 N$ points. Let

$$
X_{N}=X_{N}(a, r)=A_{N} \cup B_{N} .
$$

Define a metric $d$ on $X_{N}$ as follows: if $x, y \in A_{N}$ or $x, y \in B_{N}$, let $d(x, y)=\|x-y\|$, the straight-line distance between the two points. If $x=\left(x_{1}, 0\right) \in A_{N}$ and $y=$ $\left(y_{1}, a r^{2 N+p}\right)$, define $d(x, y)=\left|x_{1}\right|+\left|y_{1}\right|+a r^{2 N+p}$.

In any case, $d(x, y)$ can be thought of as the 'walking distance' between the two points if one is restricted to walking along the $y$-axis or the lines $y=0$ and $y=a r^{2 N+p}$. Note that for any $x, y \in X_{N}, d(x, y) \geq a$.

Define $T=T_{N}$ on $X_{N}$ as follows:

$$
\begin{aligned}
& T((0,0))=T((a, 0))=(0,0) \\
& T\left(\left(a r^{k}, 0\right)\right)=\left(-a r^{N+k-1}, 0\right) \text { for } 1 \leq k \leq N \\
& T\left(\left(-a r^{N+k}, 0\right)\right)=\left(a r^{k}, 0\right) \text { for } 0 \leq k \leq N \\
& T\left(\left(a r^{k}, a r^{2 N+p}\right)\right)=\left(a r^{k-1}, 0\right) \text { for } 1 \leq k \leq N \\
& T\left(\left(-a r^{N+k}, a r^{2 N+p}\right)\right)=\left(-a r^{N+k-1}, 0\right) \text { for } 1 \leq k \leq N .
\end{aligned}
$$

We make the following observations. 
$1^{\circ}$. If $x, y \in B_{N}, d(T x, T y) \leq(1 / r) d(x, y)$.

$2^{\circ}$. Let $p$ be the smallest integer such that $r^{p-1}>2$. If $x \in A_{N}, y \in B_{N}$, then $d(x, y) \geq a r^{2 N+p}$ and $d(T x, T y) \leq d(T x,(0,0))+d((0,0), T y) \leq 2 a r^{2 N}$, so $d(T x, T y) / d(x, y) \leq 2 a r^{2 N} / a r^{2 N+p}=2 / r^{p}<1 / r$, since $r^{p-1}>2$.

$3^{\circ}$. If $x, y \in A_{N}$, we examine $d\left(T^{2} x, T^{2} y\right)$ by looking at separate cases.

(a) if the abscissa of $x$ is non-positive and the abscissa of $y$ is non-negative, the same can be said of both $T^{2} x$ and $T^{2} y$. Since $d\left(T^{2} x,(0,0)\right) \leq(1 / r) d(x,(0,0))$, and $d\left(T^{2} y,(0,0)\right) \leq(1 / r) d(y,(0,0))$, we see that $d\left(T^{2} x, T^{2} y\right) \leq(1 / r) d(x, y)$.

(b) if the abscissas of both $x$ and $y$ are non-positive and both $T^{2} x$ and $T^{2} y$ lie to the left of the origin, then $d\left(T^{2} x, T^{2} y\right) \leq(1 / r) d(x, y)$. Suppose we assume that $T^{2} x=0$. If $x=(0,0)$, then $d\left(T^{2} x, T^{2} y\right) \leq(1 / r) d(x, y)$. If $x=\left(-a r^{N}, 0\right)$, then if $y=\left(-a r^{N+k}, 0\right)$ for $k \geq 1$, then

$$
d\left(T^{2} x, T^{2} y\right) / d(x, y)=a r^{N+k-1} /\left(a r^{N+k}-a r^{N}\right)=r^{k-1} /\left(r^{k}-1\right) .
$$

As $r \rightarrow \infty$, this fraction converges to 0 , and so, by making $r$ large we can guarantee that $d\left(T^{2} x, T^{2} y\right) / d(x, y)<1 / R$ for all such $x$ and $y$. It helps here that $X_{N}$ is a finite set.

(c) A similar argument to that presented in (b) can be given to show that, for large $r$, the ratio $d\left(T^{2} x, T^{2} y\right) / d(x, y)<1 / R$ if the abscissas of both $x$ and $y$ are non-negative. Combining $1^{\circ}, 2^{\circ}$, and $3^{\circ}$, we see that if $x, y \in X_{N}$ then

$$
\min \left\{d(T x, T y), d\left(T^{2} x, T^{2} y\right)\right\} \leq(1 / R) d(x, y) .
$$

$4^{\circ}$. Let $x=\left(a r^{N-1}, 0\right), y=\left(a r^{N}, 0\right)$. Then

$$
\min \left\{d(T x, T y), d\left(T^{3} x, T^{3} y\right), \ldots, d\left(T^{2 N-1} x, T^{2 N-1} y\right)\right\} \geq d(x, y) .
$$

5. Let $x=\left(a r^{N-1}, a r^{2 N+p}\right), y=\left(a r^{N}, a r^{2 N+p}\right)$. Then

$$
\min \left\{d\left(T^{2} x, T^{2} y\right), d\left(T^{4} x, T^{4} y\right), \ldots, d\left(T^{2 N-2} x, T^{2 N-2} y\right)\right\} \geq d(x, y) .
$$

$4^{\circ}$ and $5^{\circ}$ show that none of the maps $T^{k}$ are contractions for $1 \leq k \leq 2 N-1$.

$6^{\circ}$. Note that for any $x \in X_{N}$, since $(0,0)$ is a fixed point of $T$,

$$
\min \left\{d(T x,(0,0)), d\left(T^{2} x,(0,0)\right)\right\} \leq(1 / R) d(x,(0,0)) .
$$

We now use these spaces to construct $X$ and $T$. Assume that $M>0$ is given. First, choose $R$ such that $R>1 / M$; then $R M-1>0$. We now inductively construct $\left\{a_{n}\right\}_{n=1}^{\infty}$ and $\left\{r_{n}\right\}_{n=1}^{\infty}$.

Let $a_{1}=1$, and choose $r_{1}$ appropriately (in line with $3^{\circ}$ above). Let $Y_{1}=X_{1}\left(a_{1}, r_{1}\right)$. Assume that $Y_{k}=X_{k}\left(a_{k}, r_{k}\right)$ have been defined for $1 \leq k \leq n$. Let $s_{k}$ be the diameter of $Y_{k}$ for $1 \leq k \leq n$, and let $s=\max \left\{s_{1}, \ldots, s_{n}\right\}$. Since $R M-1>0$, let

$$
a_{n+1}=\max \{2 s, s R(1+M) /(R M-1)\} .
$$


Now choose $r_{n+1}$ to correspond to $a_{n+1}$ as in $3^{\circ}$. Let $Y_{n+1}=X_{n+1}\left(a_{n+1}, r_{n+1}\right)$.

Assume that $T_{n}$ is the map of $Y_{n}$ defined earlier. Let $H$ be separable Hilbert space, and $\left\{e_{n}: n \in \mathbf{N}\right\}$ a countable orthonormal basis for $H$. Let $S_{n}$ be the 2-dimensional subspace of $H$ generated by $e_{2 n-1}$ and $e_{2 n}$. The map $(x, y) \mapsto x e_{2 n-1}+y e_{2 n}$ maps the plane $\mathbf{R}^{2}$ isometrically onto $S_{n}$; let $W_{n}$ be the image of $Y_{n}$ under this map. Notice that the origin $O$ in $H$ is the intersection of all the $W_{n}$; we let $X$ denote the union of the $W_{n}$.

We can extend the idea of 'walking' in $X$ as follows: given two points $x, y \in X$, assume $x \in W_{k}$ and $y \in W_{n}$. Walk from $x$ to $O$ in the shortest fashion along the images of the $y$-axis and the two lines defining $Y_{k}$; then walk from $O$ to $y$ in the same manner in $W_{n} . D(x, y)$, the distance from $x$ to $y$, is the length of this walk.

Because of the requirement that $a_{n+1} \geq 2 s$, we see that $X$ is complete; as it is the union of finite sets $W_{n}$ such that each point (except $O$ ) belonging to $W_{n}$ is at distance $>a_{1}$ from any point in any other $W_{k} . T$ is defined in the obvious fashion; $T(O)=O$, and if $x \in W_{n}$, then $x$ is the image of some point $u$ in $Y_{n}$ under the canonical map, and $T x$ is the image of $T_{n} u$.

We now show $T$ satisfies (2) and (3), and the example will be complete. Since $T^{k}$ is not a contraction on $W_{n}$ for $1 \leq k \leq 2 n-1$, we see that no power of $T$ is a contraction.

Let $x, y \in X$. If $x$ and $y$ belong to the same $W_{n}$, by construction and by choice of $r_{n}$,

$$
\min \left\{D(T x, T y), D\left(T^{2} x, T^{2} y\right)\right\} \leq(1 / R) D(x, y) \leq M D(x, y) .
$$

Without loss of generality, assume that $x \in W_{n+1}$, and $y \in W_{k}$ for some $k \leq n$. We can also assume that neither of these points is $O$.

We have observed that we can choose $j$ equal to either 1 or 2 such that $D\left(T^{j} x, O\right)$ $\leq(1 / R) D(x, O)$. Since $x \in W_{n+1}$ and $y \in W_{k}$ for some $k \leq n$,

$$
D(x, O) \leq D(x, y)+D(y, O) \leq D(x, y)+s .
$$

Therefore $D(x, y) \geq D(x, O)-s$. We also have

$$
D\left(T^{j} x, T^{j} y\right) \leq D\left(T^{j} x, O\right)+D\left(O, T^{j} y\right) \leq(1 / R) D(x, O)+s .
$$

Therefore $D\left(T^{j} x, T^{j} y\right) / D(x, y) \leq((1 / R) D(x, O)+s) /(D(x, O)-s)$. Since

$$
D(x, O) \geq a_{n+1} \geq s R(1+M) /(R M-1),
$$

we see that $R M D(x, O)-D(x, O) \geq s R+s R M$, and so $D(x, O)+s R \leq$ $R M D(x, O)-s R M$. Hence we get that $((1 / R) D(x, O)+s) /(D(x, O)-s) \leq M$. Consequently, $D\left(T^{j} x, T^{j} y\right) / D(x, y) \leq M$, establishing (2). 


\section{The Generalized Banach Contraction Conjecture}

The standard proof of the Banach Contraction Principle (GBCC for $J=\{1\}$ ) involves showing that a sequence of iterates is Cauchy. Then, since contractions are continuous, it is easy to show that the limit of the sequence of iterates is a fixed point.

Of course, it is not in general true that the limit of a convergent sequence of iterates is a fixed point; a simple counterexample on $[0,1]$ can be obtained by defining $T x=x / 2$ for $x>0, T 0=1$. We mentioned in the Introduction that the hypothesis (1) in GBCC does not imply that the map satisfying (1) is continuous.

THEOREM 1. Let $(X, d)$ be a complete metric space, $0<M<1$, and let $p$ be an integer. Let $T$ be a self-map of $X$. Assume that, given $p$ pairs $\left(x_{1}, y_{1}\right), \ldots,\left(x_{p}, y_{p}\right)$ of points from $X$, for some $k, 1 \leq k \leq 2 p$, we have

$$
d\left(T^{k} x_{j}, T^{k} y_{j}\right) \leq M d\left(x_{j}, y_{j}\right), \quad \text { for } 1 \leq j \leq p .
$$

Then T has a fixed point.

Proof. Let $x_{0} \in X$, and let $[z]$ denote the greatest integer less than or equal to $z$. The proof consists of two steps. The first of these steps is to show that the hypothesis guarantees that $\left\{T^{n} x_{0}\right\}_{n=0}^{\infty}$ is Cauchy. The second step will be to show that the limit of this Cauchy sequence is a fixed point of $T$. However, because we can prove a more general result than the second step which has implications for GBCC, we shall instead prove that more general result. Let

$$
\begin{aligned}
& C_{1}=\max \left\{d\left(T^{2 k} x_{0}, T^{2 k+1} x_{0}\right): k=0,1, \ldots, p-1\right\}, \\
& C_{2}=\max \left\{d\left(T^{2 k} x_{0}, T^{2 k+2} x_{0}\right): k=0,1, \ldots, p-1\right\} .
\end{aligned}
$$

By applying the hypothesis to the $p$ pairs $\left(x_{0}, T x_{0}\right),\left(T^{2} x_{0}, T^{3} x_{0}\right), \ldots,\left(T^{2 p-2} x_{0}\right.$, $\left.T^{2 p-1} x_{0}\right)$, we obtain an integer $i_{1}$ with $1 \leq i_{1} \leq 2 p$ and such that

$$
d\left(T^{i_{1}+2 k} x_{0}, T^{i_{1}+2 k+1} x_{0}\right) \leq M C_{1}, \quad \text { for } \quad 0 \leq k \leq p-1 .
$$

Continuing, we can find a sequence $\left\{i_{n}\right\}$ such that $i_{n}+1 \leq i_{n+1} \leq i_{n}+2 p$, and

$$
d\left(T^{i_{n}+2 k} x_{0}, T^{i_{n}+2 k+1} x_{0}\right) \leq M^{n} C_{1}, \text { for } 0 \leq k \leq p-1 .
$$

By applying the hypothesis to the $p$ pairs $\left(x_{0}, T^{2} x_{0}\right),\left(T^{2} x_{0}, T^{4} x_{0}\right), \ldots,\left(T^{2 p-2} x_{0}\right.$, $T^{2 p} x_{0}$ ), we obtain an integer $j_{1}$ with $1 \leq j_{1} \leq 2 p$ and such that

$$
d\left(T^{j_{1}+2 k} x_{0}, T^{j_{1}+2 k+2} x_{0}\right) \leq M C_{2}, \text { for } 0 \leq k \leq p-1 .
$$


Continuing, we can find a sequence $\left\{j_{n}\right\}$ such that $j_{n}+1 \leq j_{n+1} \leq j_{n}+2 p$, and

$$
d\left(T^{j_{n}+2 k} x_{0}, T^{j_{n}+2 k+2} x_{0}\right) \leq M^{n} C_{2}, \quad \text { for } \quad 0 \leq k \leq p-1 .
$$

Now let $q$ be a positive integer. Since $i_{n+1} \leq i_{n}+2 p$, for some integer $r, i_{r} \leq q<$ $i_{r}+2 p$. The spacing of the integers in $\left\{i_{n}\right\}$ guarantees that $r \geq[q / 2 p]$. Similarly, for some integer $s, j_{s} \leq q<j_{s}+2 p$, and the spacing of the integers in $\left\{j_{n}\right\}$ guarantees that $s \geq[q / 2 p]$. In order to obtain a bound for $d\left(T^{q} x_{0}, T^{q+1} x_{0}\right)$, we examine three separate cases.

Case I. The integer $q$ has the form $q=i_{r}+2 k$ for some $k$ with $0 \leq k \leq p-1$. In this case,

$$
d\left(T^{q} x_{0}, T^{q+1} x_{0}\right) \leq M^{r} C_{1} \leq M^{[q / 2 p]} C_{1} .
$$

Case II. The integer $q$ has the form $q=i_{r}+2 k+1$ for some $k$ with $0 \leq k \leq p-1$, and $q$ also has the form $j_{s}+2 m+1$ with $0 \leq m \leq p-1$. In this case,

$$
\begin{aligned}
d\left(T^{q} x_{0}, T^{q+1} x_{0}\right) & \leq d\left(T^{q} x_{0}, T^{q-1} x_{0}\right)+d\left(T^{q-1} x_{0}, T^{q+1} x_{0}\right) \\
& \leq M^{r} C_{1}+M^{s} C_{2} \leq M^{[q / 2 p]}\left(C_{1}+C_{2}\right) .
\end{aligned}
$$

Case III. The integer $q$ has the form $q=i_{r}+2 k+1$ for some $k$ with $0 \leq k \leq p-1$, and $q$ also has the form $j_{s}+2 m$ with $0 \leq m \leq p-1$. If $k<p-1$, then

$$
\begin{aligned}
d\left(T^{q} x_{0}, T^{q+1} x_{0}\right) & \leq d\left(T^{q} x_{0}, T^{q+2} x_{0}\right)+d\left(T^{q+2} x_{0}, T^{q+1} x_{0}\right) \\
& \leq M^{s} C_{2}+M^{r} C_{1} \leq M^{[q / 2 p]}\left(C_{1}+C_{2}\right) .
\end{aligned}
$$

If $k=p-1$, then either $q+1=i_{r+1}$ or $i_{r+1}<q+1<i_{r+1}+2 p-1$. If $q+1=i_{r+1}$, then

$$
\begin{aligned}
d\left(T^{q} x_{0}, T^{q+1} x_{0}\right) & \leq d\left(T^{q} x_{0}, T^{q+2} x_{0}\right)+d\left(T^{q+2} x_{0}, T^{q+1} x_{0}\right) \\
& \leq M^{s} C_{2}+M^{r+1} C_{1} \leq M^{[q / 2 p]}\left(M C_{1}+C_{2}\right) \leq M^{[q / 2 p]}\left(C_{1}+C_{2}\right)
\end{aligned}
$$

If $i_{r+1}<q+1 \leq i_{r+1}+2 p-1$, then either $q=i_{r+1}+2 m$ with $0 \leq m \leq p-1$ or $q=i_{r+1}+2 m+1$ with $0 \leq m \leq p-2$. If $q=i_{r+1}+2 m$ with $0 \leq m \leq p-1$, then

$$
d\left(T^{q} x_{0}, T^{q+1} x_{0}\right) \leq M^{r+1} C_{1} \leq M^{r} C_{1} \leq M^{[q / 2 p)} C_{1} .
$$

If $q=i_{r+1}+2 m+1$ with $0 \leq m \leq p-2$, then

$$
\begin{aligned}
d\left(T^{q} x_{0}, T^{q+1} x_{0}\right) & \leq d\left(T^{q} x_{0}, T^{q+2} x_{0}\right)+d\left(T^{q+2} x_{0}, T^{q+1} x_{0}\right) \\
& \leq M^{s} C_{2}+M^{r+1} C_{1} \leq M^{[q / 2 p]}\left(M C_{1}+C_{2}\right) \leq M^{[q / 2 p]}\left(C_{1}+C_{2}\right) .
\end{aligned}
$$


In any of these cases, since $[q / 2 p] \geq(q / 2 p)-1$, we see that

$$
d\left(T^{q} x_{0}, T^{q+1} x_{0}\right) \leq M^{[q / 2 p]}\left(C_{1}+C_{2}\right) \leq C K^{q},
$$

where $K=M^{1 / 2 p}$ and $C=\left(C_{1}+C_{2}\right) / M$. Since $0<K<1$, we conclude that the sequence $\left\{T^{n} x_{0}\right\}_{n=1}^{\infty}$ forms a Cauchy sequence.

If $T$ were assumed to be continuous, a standard argument would show that the limit of the Cauchy sequence $\left\{T^{n} x_{0}\right\}_{n=1}^{\infty}$ is a fixed point. However, as we shall show, the hypothesis of GBCC is all that is needed to show that the limit is a fixed point, provided that for some integer $q,\left\{T^{k q} x_{0}\right\}_{k=1}^{\infty}$ is Cauchy, and the integer $q$ is independent of the choice of the starting point $x_{0}$.

In the proofs in Jachymski-Schröder-Stein [8] of GBCC for $J=\{1,2\},\{1,3\}$, and $\{2,3\}$, it is shown that there is a power of $T$, say $T^{q}$, such that for any point $x_{0}$, the sequence of iterates $\left\{T^{k q} x_{0}\right\}_{k=1}^{\infty}$ is Cauchy.

Assume now that (1) holds for some finite set $J$, and suppose that we can demonstrate the existence of a Cauchy sequence of iterates of some power of $T$. Moreover, assume that this power of $T$ depends only on the set $J$ that appears in (1), and not the point used to start the iteration. We now show that $T$ has a fixed point. This will eliminate the necessity of assuming that $T$ is continuous.

For the purpose of this argument, assume that $N$ is an integer such that $\left\{T^{k N} x\right\}_{k=1}^{\infty}$ is Cauchy for any $x \in X$. Now pick a specific $x_{0} \in X$. For any $j \geq 0$, since $X$ is complete, assume that the sequence $\left\{T^{k N+j} x_{0}\right\}_{k=1}^{\infty}$ converges to $z_{j}$. Since $\left\{T^{k N+j+N} x_{0}\right\}_{k=1}^{\infty}$ is a subsequence of $\left\{T^{k N+j} x_{0}\right\}_{k=1}^{\infty}$, we see that $z_{j+N}=z_{j}$. In other words, there are at most $N$ distinct limits among $\left\{z_{j}: j=0,1,2, \ldots\right\}$. Let $z=z_{0}$.

Apply (1) to the sequence of pairs $\left(T^{k N} x, z\right)$. Since $J$ is finite, for some $j_{1} \in J$, $d\left(T^{k N+j_{1}} x, T^{j_{1}} z\right) \leq M d\left(T^{k N} x, z\right)$, for infinitely many $k$. Since $T^{k N} x \rightarrow z$, this implies there exists a subsequence $\left\{k_{n}^{(1)}\right\}$ such that $T^{k_{n}^{(1)} N+j_{1}} x \rightarrow T^{j^{\prime}} z$. Now apply (1) to the sequence of pairs $\left(T^{k_{n}^{(1)} N+j_{1}} x, T^{j_{1}} z\right)$. Arguing as above, we can find an integer $j_{2} \in J$ and a subsequence $\left\{k_{n}^{(2)}\right\}$ of $\left\{k_{n}^{(1)}\right\}$ such that

$$
T^{k_{n}^{(2)} N+j_{1}+j_{2}} x \rightarrow T^{j_{1}+j_{2}} z
$$

Now apply (1) to the sequence of pairs $\left(T^{k_{n}^{(2)} N+j_{1}+j_{2}} x, T^{j_{1}+j_{2}} z\right)$, and continue.

Re-labeling terms appropriately, we thus arrive at the matrix $\left\{x_{k, n}: n \in \mathbf{N}, k \in \mathbf{N}\right\}$ with the following properties:

each $x_{k, n}$ has the form $T^{j N} x_{0}$;

$\left\{x_{k, n}\right\}_{n=1}^{\infty}$ is a subsequence of $\left\{x_{k-1, n}\right\}_{n=1}^{\infty}$ for $k>1$;

$$
T^{j_{1}+\cdots+j_{k}} x_{k, n} \rightarrow T^{j_{1}+\cdots+j_{k}} z \quad \text { as } n \rightarrow \infty .
$$

However, as we observed two paragraphs ago, the expression on the left side of (4) depends only on the residue class $\bmod N$ of $j_{1}+\cdots+j_{k}$. Sooner or later, we will find 
integers $q>p$ such that $j_{1}+\cdots+j_{q}$ and $j_{1}+\cdots+j_{p}$ belong to the same residue class $\bmod N$. Therefore, if $\alpha=j_{1}+\cdots+j_{q}$ and $\beta=j_{1}+\cdots+j_{p}$, then $T^{\alpha} z=T^{\beta} z$, and so $T^{\alpha-\beta}\left(T^{\beta} z\right)=T^{\beta} z$. So $T$ has a periodic point.

The same type of argument can be used to show that the periodic point is actually a fixed point. Suppose that $T$ has a periodic point $u$ of order $M$. Apply (1) repetitively to the pair $(T u, u)$. We obtain a sequence of pairs $\left(T^{k_{n}+1} u, T^{k_{n}} u\right)$, where the exponents $k_{n}$ are taken from $Z_{M}$, the integers $\bmod M$. These pairs have the following property:

$$
d\left(T^{k_{n+1}+1} u, T^{k_{n+1}} u\right) \leq M d\left(T^{k_{n}+1} u, T^{k_{n}} u\right)
$$

Since $Z_{M}$ is finite, there must be integers $p>n$ such that $k_{p}=k_{n}$, and for these integers we have

$$
d\left(T^{k_{p}+1} u, T^{k_{p}} u\right) \leq M^{p-n} d\left(T^{k_{n}+1} u, T^{k_{n}} u\right) .
$$

Since $M^{p-n}<1, T^{k_{n}+1} u=T^{k_{n}} u$. If $0 \leq k_{n} \leq M-2$, then the previous equality shows that $T^{k_{n}} u$ is a fixed point of $T$. If $k_{n}=M-1$, then $u=T^{M} u=T^{M-1} u$, and so $T^{M-1} u$ is a fixed point of $T$.

We remark that the above theorem holds if, given $p+1$ pairs $\left(x_{1}, y_{1}\right), \ldots,\left(x_{p+1}\right.$, $\left.y_{p+1}\right)$ of points from $X$, for some $k, 1 \leq k \leq 2 p+1$, we have $d\left(T^{k} x_{j}, T^{k} y_{j}\right)$ $\leq M d\left(x_{j}, y_{j}\right)$ for $1 \leq j \leq p+1$. This assumption would imply that for some $k$, $1 \leq k \leq 2 p+2$, we have $d\left(T^{k} x_{j}, T^{k} y_{j}\right) \leq M d\left(x_{j}, y_{j}\right)$ for $1 \leq j \leq p+1$, and we could then apply Theorem 1 .

In the second part of the proof of Theorem 1 we have proved in fact the following result.

Proposition 1. Let $(X, d)$ be a complete metric space, $0<M<1$, and let $N$ be a positive integer. Let $T$ be a self-map of $X$ such that $T$ satisfies (1) for some finite set $J$. If for any $x \in X$ the sequence $\left\{T^{k N} x\right\}_{k=1}^{\infty}$ is Cauchy, then $T$ has a fixed point.

The next theorem demonstrates that there are hypotheses related to (1) which result in fixed points for any finite set $J$.

THEOREM 2. Let $(X, d)$ be a complete metric space, and assume that $T: X \mapsto X$ is uniformly continuous. Let $u$ be an upper semi-continuous function defined on the non-negative reals such that $u(t)<t$ for all $t>0$, and $\lim _{t \rightarrow \infty} \inf (t-u(t))>0$. Let $N$ be a positive integer. Assume that for all $x, y \in X$

$$
\min \left\{d(T x, T y), d\left(T^{2} x, T^{2} y\right), \ldots, d\left(T^{N} x, T^{N} y\right)\right\} \leq u(d(x, y)) .
$$

Then T has a unique fuxed point. 
PROOF. Choose a positive integer $p$ such that $\lim _{t \rightarrow \infty} \inf (t-u(t))>2 / p$. For each $n \in \mathbf{N}$, let

$$
A_{n}=\bigcap_{j=1}^{n}\left\{x \in X: d\left(x, T^{j} x\right) \leq 1 /(n+p)\right\} .
$$

Let $F(T)$ denote the set of all fixed points of $T$; it is clear that $F(T)=\cap_{n=1}^{\infty} A_{n}$. In order to prove that $T$ has a unique fixed point we must show that $F(T)$ is a singleton. To do so, we show that the $\left\{A_{n}: n=1,2, \ldots\right\}$ form a decreasing sequence of non-empty closed sets whose diameters converge to 0 ; the result then follows by the Cantor Intersection Theorem. The sets form a decreasing sequence since $\{1 /(n+p)\}_{n=1}^{\infty}$ is a decreasing sequence of real numbers, and each $A_{n}$ is closed because it is the intersection of the inverse images of the closed interval $[0,1 /(n+p)]$ under the continuous maps $x \mapsto d\left(x, T^{j} x\right)$.

It remains to show that the $A_{n}$ are non-empty, and that their diameters converge to 0 . Let $\alpha=\inf \{d(x, T x): x \in X\}$. Choose a sequence $\left\{x_{n}\right\}_{n=1}^{\infty}$ such that $d\left(x_{n}, T x_{n}\right) \rightarrow \alpha^{+}$. Letting $x=x_{n}$ and $y=T x_{n}$ in (5), we see that, for all positive integers $n$,

$$
\begin{aligned}
\alpha & \leq \min \left\{d\left(T x_{n}, T\left(T x_{n}\right)\right), d\left(T^{2} x_{n}, T\left(T^{2} x_{n}\right)\right), \ldots, d\left(T^{N} x_{n}, T\left(T^{N} x_{n}\right)\right)\right\} \\
& \leq u\left(d\left(x_{n}, T x_{n}\right)\right) .
\end{aligned}
$$

Letting $n \rightarrow \infty$, we see that

$$
\alpha \leq \lim _{n \rightarrow \infty} \sup u\left(d\left(x_{n}, T x_{n}\right)\right) \leq \lim _{t \rightarrow \alpha^{+}} \sup u(t) \leq u(\alpha)
$$

since $u$ is upper semi-continuous. By assumption, $u(t)<t$ for $t>0$, so $\alpha=0$.

Since $T$ is uniformly continuous, all the maps $T^{i}$ are uniformly continuous, and so given $\epsilon>0$, there is $\delta_{\epsilon}>0$ such that $d(x, y)<\delta_{\epsilon}$ implies $d\left(T^{i} x, T^{i} y\right)<\epsilon$ for $1 \leq i \leq N-1$; and we may assume without loss of generality that $\delta_{\epsilon}<\epsilon / N$. Now let $\epsilon>0$. Since $\inf \{d(x, T x): x \in X\}=0$, there exists an $x_{\epsilon} \in X$ such that $d\left(x_{\epsilon}, T x_{\epsilon}\right)<\delta_{\epsilon}$. Then, for $1 \leq j \leq N$,

$$
d\left(x_{\epsilon}, T^{j} x_{\epsilon}\right) \leq \sum_{i=0}^{j-1} d\left(T^{i} x_{\epsilon}, T^{i+1} x_{\epsilon}\right) \leq \sum_{i=0}^{N-1} d\left(T^{i} x_{\epsilon}, T^{i+1} x_{\epsilon}\right)<\delta_{\epsilon}+(N-1) \epsilon / N<\epsilon .
$$

Letting $\epsilon=1 /(n+p)$, we see that $x_{\epsilon} \in A_{n}$, and so $A_{n}$ is non-empty.

To complete the proof we show that the diameters of the $A_{n}$ converge to 0 . Let $a_{n}=\operatorname{diam}\left(A_{n}\right) ; a_{n}$ is possibly infinite. Let $x, y \in A_{n}$, and choose an integer $j \leq N$ such that $d\left(T^{j} x, T^{j} y\right) \leq u(d(x, y))$. Then

$$
d(x, y) \leq d\left(x, T^{j} x\right)+d\left(T^{j} x, T^{j} y\right)+d\left(T^{j} y, y\right) \leq u(d(x, y))+2 /(n+p) .
$$


We therefore see that $d(x, y)-u(d(x, y)) \leq 2 /(n+p)$ for any $x, y$ belonging to $A_{n}$.

Suppose that $a_{n}$ is infinite for some $n$. Then we can find sequences $\left\{x_{n}\right\}_{n=1}^{\infty}$ and $\left\{y_{n}\right\}_{n=1}^{\infty}$ in $A_{n}$ such that $t_{k}=d\left(x_{k}, y_{k}\right) \rightarrow \infty$. But then $t_{k}-u\left(t_{k}\right) \leq 2 /(n+p)$, and so $\lim _{t \rightarrow \infty} \inf (t-u(t)) \leq 2 /(n+p)<2 / p$, which contradicts the definition of $p$. Therefore each $A_{n}$ is bounded.

Since the function $t \mapsto t-u(t)$ is lower semi-continuous, $d(x, y)-u(d(x, y)) \leq$ $2 /(n+p)$ for any $x, y$ belonging to $A_{n}$ implies that $a_{n}-u\left(a_{n}\right) \leq 2 /(n+p)$. Since the sequence $\left\{A_{n}\right\}_{n=1}^{\infty}$ is decreasing, $a_{1} \geq a_{2} \geq \cdots \geq 0$; so let $a=\lim _{n \rightarrow \infty} a_{n}$. Again using the lower semi-continuity of $t \mapsto t-u(t)$, letting $n \rightarrow \infty$, we see that

$$
a-u(a) \leq \lim _{t \rightarrow \infty} \inf (t-u(t)) \leq \lim _{n \rightarrow \infty} \inf \left(a_{n}-u\left(a_{n}\right)\right) \leq 0 .
$$

Therefore $a \leq u(a)$, and so $a=0$. Hence $\operatorname{diam}\left(A_{n}\right) \rightarrow 0$, and Cantor's Intersection Theorem shows that $F(T)$ is a singleton.

Hypothesis (5) in Theorem 1 is a hybrid of two ideas: the hypothesis (1) of GBCC and a theorem of Browder [1].

We recall the following definition introduced by Browder and Petryshyn [2].

DEFINITION 1. A map T is asymptotically regular if for each $x \in X, \lim _{n \rightarrow \infty} d\left(T^{n} x\right.$, $\left.T^{n+1} x\right)=0$.

The next theorem makes use of the following result of Dugundji ([5, pp. 13-14] or [4]).

THEOREM 3 (Dugundji). Let $(X, d)$ be a complete metric space, $\alpha: X \mapsto \mathbf{R}_{+}$an arbitrary function. Assume that if $a>0$,

$$
\gamma(a)=\inf \{\alpha(x)+\alpha(y): d(x, y) \geq a\}>0 .
$$

Then any sequence $\left\{x_{n}\right\}$ for which $\alpha\left(x_{n}\right) \rightarrow 0$, converges to the same point.

THEOREM 4. Let $(X, d)$ be complete, and assume that $T: X \mapsto X$. Let $u$ satisfy conditions of Theorem 2 , and assume that $T$ satisfies condition (5). Then $T$ has a contractive fixed point (a fixed point to which every sequence of iterates $T^{n} x$ converges) if and only if $T$ is asymptotically regular.

PROOF. Note that $T$ is not assumed to be continuous. The 'only if' part of the theorem is trivial, so assume that $T$ is asymptotically regular. Define $\alpha: X \mapsto \mathbf{R}_{+}$by

$$
\alpha(x)=\max \left\{d\left(x, T^{j} x\right): 1 \leq j \leq N\right\} .
$$


Assume that $\alpha$ does not satisfy the conditions of Dugundji's Theorem. Then there exists an $\epsilon_{0}>0$ and sequences $\left\{x_{n}\right\}$ and $\left\{y_{n}\right\}$ such that $\alpha\left(x_{n}\right)+\alpha\left(y_{n}\right)<1 / n$ and $t_{n}=d\left(x_{n}, y_{n}\right) \geq \epsilon_{0}$.

Observe that, for any $k \in\{1,2, \ldots, N\}$,

$$
d\left(x_{n}, y_{n}\right) \leq d\left(x_{n}, T^{k} x_{n}\right)+d\left(T^{k} x_{n}, T^{k} y_{n}\right)+d\left(T^{k} y_{n}, y_{n}\right),
$$

so choosing $k$ in accordance with condition (5) we have

$$
d\left(x_{n}, y_{n}\right) \leq \alpha\left(x_{n}\right)+\alpha\left(y_{n}\right)+u\left(d\left(x_{n}, y_{n}\right)\right)<1 / n+u\left(d\left(x_{n}, y_{n}\right)\right),
$$

and so $t_{n}-u\left(t_{n}\right)<1 / n$. If $\left\{t_{n}\right\}$ is not bounded, then $\lim _{t \rightarrow \infty} \inf (t-u(t))=$ 0 , a contradiction. So we can assume without loss of generality, by passing to a subsequence if necessary, that $t_{n} \rightarrow t_{0}$. Combining the inequality $t_{n}-u\left(t_{n}\right)<1 / n$ with the semi-continuity of $u$, and letting $n \rightarrow \infty$ shows that $t_{0} \leq u\left(t_{0}\right)$. Therefore $t_{0}=0$, contradicting the fact that $t_{n} \geq \epsilon_{0}$, and so $\alpha$ satisfies the hypotheses of Dugundji's Theorem.

Note that, for some $k \in\{1,2, \ldots, N\}$,

$$
\alpha\left(T^{n} x\right)=d\left(T^{n} x, T^{n+k} x\right) \leq \sum_{i=0}^{k-1} d\left(T^{n+i} x, T^{n+i+1} x\right) \leq \sum_{i=0}^{N-1} d\left(T^{n+i} x, T^{n+i+1} x\right),
$$

and this last expression approaches 0 as $n \rightarrow \infty$ by asymptotic regularity. So by Dugundji's Theorem, $T^{n} x \rightarrow z$, where $z$ does not depend on $x$. By an earlier argument involving a condition similar to (5) and the existence of a limit independent of the start of the sequence, $z=T z$.

COROLlaRY 1. Assume the hypotheses of Theorem 4 are satisfied with $N=2$. Then $T$ has a contractive fixed point.

PROOF. From Theorem 4, it is enough to show that $d\left(T^{n} x, T^{n+1} x\right) \rightarrow 0$ for $x \in X$. Assume additionally that $u$ is non-decreasing. We repeat the argument for $J=\{1,2\}$ in Jachymski-Schröder-Stein [8]. By the monotonicity of $u$, we can conclude as in Jachymski-Schröder-Stein [8] that

$$
d\left(T^{q} x, T^{g+1} x\right) \leq u^{[(q-1) / 2]}(d(x, T x))+u^{[(q-1) / 2]}\left(d\left(x, T^{2} x\right)\right) .
$$

Browder [1] has shown that $u^{n}(t) \rightarrow 0$, so $d\left(T^{n} x, T^{n+1} x\right) \rightarrow 0$.

To complete the proof, we show that if $u$ is not non-decreasing, then there is a continuous, non-decreasing function $w$ defined on $\mathbf{R}_{+}$such that

$$
u(t) \leq w(t)<t \quad \text { for } \quad t>0, \quad \lim _{t \rightarrow \infty} \inf (t-w(t))>0
$$


Then (5) is satisfied with $w$ substituted for $u$, and we may repeat the argument used in the first part of the proof.

By hypothesis, for $t>0, \lim _{s \rightarrow t} \sup u(s) \leq u(t)<t$. Hence by Jachymski [7, Theorem 2], there exists a continuous increasing function $v$ such that $u(t) \leq v(t)<t$ for all $t>0$. Moreover, by hypothesis there exist positive reals $t_{0}$ and $a$ such that $u(t) \leq t-a$ for $t \geq t_{0}$. If $v\left(t_{0}\right) \geq t_{0}-a$, then it suffices to set $w(t)=v(t)$ for $0 \leq t<t_{0}, w(t)=v\left(t_{0}\right)$ for $t_{0} \leq t \leq a+v\left(t_{0}\right)$, and $w(t)=t-a$ for $t>a+v\left(t_{0}\right)$.

So assume that $v\left(t_{0}\right)<t_{0}-a$. The set $S=\left\{t \in\left[0, t_{0}\right]: t-v(t)=a\right\}$ is closed and non-empty since $v$ is continuous, $-v(0)<a$ and $t_{0}-v\left(t_{0}\right)>a$. If $s_{0}=\max S$, then $0<s_{0}<t_{0}$ and $v(t) \leq t-a$ for $s_{0} \leq t \leq t_{0}$. So it suffices to set $w(t)=v(t)$ for $0 \leq t \leq s_{0}$ and $w(t)=t-a$ for $t>s_{0}$. It is easily seen that $w$ has all the required properties.

REMARK 1. A result similar to Theorems 2 and 4 was obtained by Wong [10, Proposition 5]: if $T$ is a nonexpansive $(d(T x, T y) \leq d(x, y)$ for $x, y \in X)$ self-map of a metric space $(X, d)$ and $T$ has a fixed point, then $T$ has a contractive fixed point if and only if there exists a function $u: \mathbf{R}_{+} \mapsto \mathbf{R}_{+}$such that $u$ is upper semi-continuous from the right, $u(t)<t$ for $t>0$ and

$$
\inf \left\{d\left(T^{n} x, T^{n} y\right): n \in \mathbf{N}\right\} \leq u(d(x, y)), \quad \text { for all } x, y \in X .
$$

Hypothesis (1) of GBCC also can be transferred into a fixed-point theorem of Caristi [3]. Part of the utility of Caristi's Theorem is that the self-map $T$ need not be continuous. However, the proof in the case where $T$ is continuous is much simpler, and since the purpose of this paper is to investigate variations of $(1)$ in different settings, we shall assume that $T$ is continuous. As usual, $(X, d)$ is a complete metric space.

THEOREM 5. Suppose that $\alpha: X \mapsto \mathbf{R}_{+}$is such that, for each $x \in X$ and $k=1,2$; either

$$
d\left(x, T^{k} x\right) \leq \alpha(x)-\alpha\left(T^{k} x\right)
$$

or

$$
d\left(T x, T^{k+1} x\right) \leq \alpha(T x)-\alpha\left(T^{k+1} x\right) .
$$

Then $T$ has a fixed point.

PROOF. Pick $x_{0} \in X$, and note that the repeated application of (6) or (7) with $k=1$ leads to an increasing sequence $\left\{i_{n}\right\}$ such that $i_{n+1} \leq i_{n}+2$, and

$$
d\left(T^{i_{n}} x_{0}, T^{i_{n}+1} x_{0}\right) \leq \alpha\left(T^{i_{n}} x_{0}\right)-\alpha\left(T^{i_{n}+1} x_{0}\right) .
$$


Similarly, repeated application of (6) or (7) with $k=2$ leads to an increasing sequence $\left\{j_{n}\right\}$ such that $j_{n+1} \leq j_{n}+2$, and also

$$
d\left(T^{j_{n}} x_{0}, T^{j_{n}+2} x_{0}\right) \leq \alpha\left(T^{j_{n}} x_{0}\right)-\alpha\left(T^{j_{n}+2} x_{0}\right)
$$

For simplicity, let $a_{n}=\alpha\left(T^{n} x_{0}\right)-\alpha\left(T^{n+1} x_{0}\right)$ and $b_{n}=\alpha\left(T^{n} x_{0}\right)-\alpha\left(T^{n+2} x_{0}\right)$. Notice that $b_{n}=a_{n}+a_{n+1}$.

We estimate $d\left(T^{q} x_{0}, T^{q+1} x_{0}\right)$. If $q=i_{n}$ for some $n$, then $d\left(T^{q} x_{0}, T^{q+1} x_{0}\right) \leq a_{q}$. If $q \notin\left\{i_{n}: n \in \mathbf{N}\right\}$, then both $q-1$ and $q+1$ belong to $\left\{i_{n}: n \in \mathbf{N}\right\}$, in which case both $d\left(T^{q-1} x_{0}, T^{q} x_{0}\right) \leq a_{q-1}$ and $d\left(T^{q+1} x_{0}, T^{q+2} x_{0}\right) \leq a_{q+1}$.

Either $q$ or $q-1 \in\left\{j_{n}: n \in \mathbf{N}\right\}$. If $q \in\left\{j_{n}: n \in \mathbf{N}\right\}$, then

$$
\begin{aligned}
d\left(T^{q} x_{0}, T^{q+1} x_{0}\right) & \leq d\left(T^{q} x_{0}, T^{q+2} x_{0}\right)+d\left(T^{q+2} x_{0}, T^{q+1} x_{0}\right) \\
& \leq b_{q}+a_{q+1}=a_{q}+2 a_{q+1} .
\end{aligned}
$$

If $q-1 \in\left\{j_{n}: n \in \mathbf{N}\right\}$, then

$$
\begin{aligned}
d\left(T^{q} x_{0}, T^{q+1} x_{0}\right) & \leq d\left(T^{q-1} x_{0}, T^{q+1} x_{0}\right)+d\left(T^{q-1} x_{0}, T^{q} x_{0}\right) \\
& \leq b_{q-1}+a_{q-1}=2 a_{q-1}+a_{q} .
\end{aligned}
$$

Therefore we always have

$$
d\left(T^{q} x_{0}, T^{q+1} x_{0}\right) \leq 2 a_{q-1}+a_{q}+2 a_{q+1} .
$$

Since $a_{1}+\cdots+a_{n}=\alpha(T x)-\alpha\left(T^{n+1} x\right) \leq \alpha(T x)$, we observe as usual that the sequence of partial sums of the series $\sum_{n=0}^{\infty} d\left(T^{n} x_{0}, T^{n+1} x_{0}\right)$ form a bounded monotone sequence. So the sequence $\left\{T^{n} x_{0}\right\}$ converges, and continuity insures that the limit of this sequence is a fixed point of $T$.

\section{Combinatorial aspects of a summation hypothesis}

The observation is made in Jachymski-Schröder-Stein [8] that there is a relation between $\mathrm{GBCC}$ and certain tiling problems in combinatorics. This relation is exploited in proving GBCC in the cases $J=\{1,2\}, J=\{1,3\}$, and $J=\{2,3\}$ by replacing the tedious and highly computational process of constructing bounds for iterates with a set of rules for manipulation of tiles.

In this section we show that other combinatorial arguments can also be useful in fixed-point theory.

Let $(X, d)$ denote a complete metric space. 
DEFINITION 2. Let $\mathscr{F}=\left\{T_{1}, \ldots, T_{N}\right\}$ be a collection of self-maps of $X$. We say that $\mathscr{F}$ has property $\sum_{p}$ if, for every set of $p$ pairs of points $\left(x_{1}, y_{1}\right), \ldots,\left(x_{p}, y_{p}\right)$, there is a $Q \in \mathscr{F}$ such that

$$
\sum_{n=1}^{\infty} d\left(Q^{n} x_{k}, Q^{n} y_{k}\right)<\infty \quad \text { for } \quad 1 \leq k \leq p .
$$

The summation hypothesis in the above definition is not as easily verified, from an a priori standpoint, as a hypothesis of contractive type, and of course this limits its applicability. However, the types of theorems obtained in this section, as well as the combinatorial aspects of the proofs, should justify investigation of this hypothesis, as the ideas may play a role in fixed-point theorems for more easily-verified hypotheses.

The idea of assuming that simultaneous (that is, for several pairs of points, rather than just one) boundedness hypotheses are satisfied has already been seen in Theorem 1. It is prompted by the fact that, in Jachymski-Schröder-Stein [8], the proof of GBCC for the case $J=\{1,2,3\}$ is tantalizingly incomplete. The remark following Theorem 1 shows that if, given any two pairs of points $(x, y)$ and $(u, v)$, there is an operator $Q \in\left\{T, T^{2}, T^{3}\right\}$ such that both $d(Q x, Q y) \leq M d(x, y)$ and $d(Q u, Q v) \leq M d(u, v)$, then $T$ has a fixed point.

We let $C(n, k)=n ! /(k !(n-k) !)$ denote the binomial coefficient. Recall that, from combinatorics, $C(n, k)$ also represents the number of subsets of $k$ items from a collection of $n$ items.

If $T:[0,1] \mapsto[0,1]$ is defined by $T 0=1, T x=x / 2$ for $x>0$, then $J=\{T\}$ satisfies $\sum_{n}$ for any $n$, but $T$ has no fixed point. $T$ is not continuous; to avoid this difficulty we will usually be forced to assume in this section that maps are continuous.

THEOREM 6. Let $q$ be a positive integer. Then there is a positive integer $N_{0}(q)$ with the following property: if $N \geq N_{0}(q)$ and $\mathscr{F}=\left\{T_{1}, \ldots, T_{N}\right\}$ consists of continuous maps and has property $\sum_{N-q}$, then some $Q \in \mathscr{F}$ has a fixed point.

ProOf. Suppose that $\mathscr{F}=\left\{T_{1}, \ldots, T_{N}\right\}$ has property $\sum_{N-q}$. Let $x_{0} \in X$, and let $I$ be a subset of $\{1,2, \ldots, N\}$ of cardinality $N-q$. By assumption, there is a $Q \in \mathscr{F}$ such that $\sum_{n=1}^{\infty} d\left(Q^{n} x_{0}, Q^{n} T_{k} x_{0}\right)<\infty$ for $k \in I$. If $Q=T_{j}$ for some $j \in I$, then the usual argument shows that $\left\{T_{j}^{n} x_{0}\right\}_{n=1}^{\infty}$ is a Cauchy sequence whose limit is a fixed point, so we can assume without loss of generality that $Q \notin\left\{T_{j}: j \in I\right\}$. Therefore, each subset $I$ of $\{1,2, \ldots, N\}$ which has cardinality $N-q$ determines an operator $Q_{I}=T_{j}$; we say that $T_{j}$ has been determined. As $I$ runs through all subsets of $\{1,2, \ldots, N\}$ with cardinality $N-q$, the number of times the operator $T_{j}$ is determined will be denoted by $\delta_{j}$. Observe that $\delta_{j} \leq C(N-1, q-1)$, because the 'without loss of generality' assumption made earlier in this paragraph requires that each time a specific operator $Q$ is determined, it is one element of a subset of 
$q=N-(N-q)$ members of $\mathscr{F}$, and the other $q-1$ members of this subset are taken from the $N-1$ members of $\mathscr{F} \backslash\{Q\}$.

Note that, since each subset $I$ of $\{1,2, \ldots, N\}$ with cardinality $N-q$ determines a member of $\mathscr{F}$, the sum $\delta_{1}+\cdots+\delta_{N}=C(N, N-q)=C(N, q)$, the number of such subsets.

We assert that, if $Q \in \mathscr{F}$ is determined $C(N-2, N-q)+1$ times, then $\sum_{n=1}^{\infty} d\left(Q^{n} x_{0}, Q^{n} T_{k} x_{0}\right)<\infty$ for $T_{k} \neq Q$. It is possible that, in choosing subsets of cardinality $N-q$ of the $N-1$ members of $\mathscr{F} \backslash\{Q\}$, after having chosen a total of $C(N-2, N-q)$ such subsets the union of these subsets may have consisted of only $N-2$ members of $\mathscr{F} \backslash\{Q\}$, but the next such subset must contain the remaining member of $\mathscr{F} \backslash\{Q\}$. Let $\gamma$ denote the number of operators $T_{j}$ for which $\delta_{j} \geq C(N-2, N-q)+1$, and let $p$ denote the largest even integer $\leq \gamma$. For notational convenience, re-index the operators so that $\delta_{j} \geq C(N-2, N-q)+1$ for $1 \leq j \leq p$. After this re-indexing, we see that $\delta_{1}+\cdots+\delta_{p} \leq p C(N-1, q-1)$.

It is possible that $\gamma$ is odd, in which case there is a single operator $T_{j}$ with $j \geq p+1$ such that $\delta_{j} \geq C(N-2, N-q)+1$, but in any case we have

$$
\delta_{p+1}+\cdots+\delta_{N} \leq C(N-1, q-1)+(N-p-1) C(N-2, N-q) .
$$

Combining this with the inequality at the end of the last paragraph, we have

$$
\delta_{1}+\cdots+\delta_{N} \leq(p+1) C(N-1, q-1)+(N-p-1) C(N-2, N-q) .
$$

We now use the above inequality to show that there is an integer $N_{0}(q)$ such that $N \geq N_{0}(q)$ implies $p \geq 2 q$. To do so, we show that, for large $N$,

$$
(p+1) C(N-1, q-1)+(N-p-1) C(N-2, N-q)<C(N, q)=\delta_{1}+\cdots+\delta_{N}
$$

for $p=0,2,4, \ldots, 2 q-2$. Note that both $(N-p-1) C(N-2, N-q)=$ $(N-p-1) C(N-2, q-2)$ and $(p+1) C(N-1, q-1)$ are polynomials of degree $q-1$ in $N$, and so their sum is also a polynomial of degree $q-1$ in $N$. But $C(N, q)$ is a polynomial of degree $q$ in $N$. Choose an integer $N_{0}(q)$ such that, for $N \geq N_{0}(q)$,

$$
(p+1) C(N-1, q-1)+(N-p-1) C(N-2, N-q)<C(N, q)=\delta_{1}+\cdots+\delta_{N}
$$

for $p=0,2,4, \ldots, 2 q-2$. This would be a contradiction unless $p \geq 2 q$.

Since $p \geq 2 q$, the set of pairs $\left(T_{1} x_{0}, T_{2} x_{0}\right), \ldots,\left(T_{p-1} x_{0}, T_{p} x_{0}\right),\left(x_{0}, T_{p+1} x_{0}\right), \ldots$, $\left(x_{0}, T_{N} x_{0}\right)$ has cardinality not greater than $N-q$, and we can now use the assumption that $\mathscr{F}$ has property $\sum_{N-q}$. We can therefore find an operator $T_{k}$ with $1 \leq k \leq N$ 
such that both

$$
\begin{aligned}
& \sum_{n=1}^{\infty} d\left(T_{k}^{n} T_{j} x_{0}, T_{k}^{n} T_{j+1} x_{0}\right)<\infty \quad \text { for } j=1,3, \ldots, p-1, \\
& \sum_{n=1}^{\infty} d\left(T_{k}^{n} x_{0}, T_{k}^{n} T_{j} x_{0}\right)<\infty \quad \text { for } j=p+1, p+2, \ldots, N .
\end{aligned}
$$

If $k \geq p+1$, by (9) we have $\sum_{n=1}^{\infty} d\left(T_{k}^{n} x_{0}, T_{k}^{n+1} x_{0}\right)<\infty$, and the usual arguments show that the sequence $\left\{T_{k}^{n} x_{0}\right\}_{n=1}^{\infty}$ is Cauchy, and the continuity of $T_{k}$ insures that the limit is a fixed point.

If $k \leq p$, let $i=k+1$ if $k$ is odd and $i=k-1$ if $k$ is even. By (8), $\sum_{n=1}^{\infty} d\left(T_{k}^{n+1} x_{0}, T_{k}^{n} T_{i} x_{0}\right)<\infty$. Since $\delta_{k} \geq C(N-2, N-q)+1$, we also have $\sum_{n=1}^{\infty} d\left(T_{k}^{n} x_{0}, T_{k}^{n} T_{i} x_{0}\right)<\infty$. Combining these two inequalities,

$$
\sum_{n=1}^{\infty} d\left(T_{k}^{n} x_{0}, T_{k}^{n+1} x_{0}\right) \leq \sum_{n=1}^{\infty} d\left(T_{k}^{n} x_{0}, T_{k}^{n} T_{i} x_{0}\right)+\sum_{n=1}^{\infty} d\left(T_{k}^{n+1} x_{0}, T_{k}^{n} T_{i} x_{0}\right)<\infty,
$$

and as before this implies that $T_{k}$ has a fixed point.

The proof of the next theorem uses some of the same ideas.

THEOREM 7. Let $\mathscr{F}=\left\{T_{1}, \ldots, T_{N}\right\}$ consist of continuous maps and have property $\sum_{N-1}$. Then some $Q \in \mathscr{F}$ has a fixed point.

Proof. Let $x_{0} \in X$. If $1 \leq k \leq N$, let $I_{k}=\{j: 1 \leq j \leq N$ and $j \neq k\}$. Since $\mathscr{F}$ has property $\sum_{N-1}$, for each $k \leq N$ we can choose $Q_{k} \in \mathscr{F}$ such that $\sum_{n=1}^{\infty} d\left(Q_{k}^{n} x_{0}, Q_{k}^{n} T_{j} x_{0}\right)<\infty$ for $j \in I_{k}$. If $Q_{k} \neq T_{k}$, then $Q_{k}$ has a fixed point via the usual argument by letting $T_{j}=Q_{k}$ in the summation at the end of the previous sentence, so as in the preceding theorem we can assume without loss of generality that $Q_{k}=T_{k}$. So

$$
\sum_{n=1}^{\infty} d\left(T_{k}^{n} x_{0}, T_{k}^{n} T_{j} x_{0}\right)<\infty \quad \text { for } \quad j \neq k, \quad 1 \leq k \leq N
$$

Now choose $Q \in \mathscr{F}$ such that $\sum_{n=1}^{\infty} d\left(Q^{n} T_{j} x_{0}, Q^{n} T_{j+1} x_{0}\right)<\infty$ for $1 \leq j \leq N-1$. Assume $Q=T_{k}$. If $k \leq N-1$, let $i=k+1$; if $k=N$, let $i=N-1$. Then $\sum_{n=1}^{\infty} d\left(T_{k}^{n+1} x_{0}, T_{k}^{n} T_{i} x_{0}\right)<\infty$. Since $i \neq k$ we also have $\sum_{n=1}^{\infty} d\left(T_{k}^{n} x_{0}, T_{k}^{n} T_{i} x_{0}\right)<$ $\infty$. As in the preceding theorem, we see that

$$
\sum_{n=1}^{\infty} d\left(T_{k}^{n} x_{0}, T_{k}^{n+1} x_{0}\right) \leq \sum_{n=1}^{\infty} d\left(T_{k}^{n} x_{0}, T_{k}^{n} T_{i} x_{0}\right)+\sum_{n=1}^{\infty} d\left(T_{k}^{n+1} x_{0}, T_{k}^{n} T_{i} x_{0}\right)<\infty,
$$

and as usual this implies that $T_{k}$ has a fixed point. 
COROLLARY 2. Let $S$ and $T$ be continuous self-maps of $X$, and let $p$ be a positive integer. Assume that $\mathscr{F}=\left\{S, S^{2}, \ldots, S^{p}, T, T^{2}, \ldots, T^{p}\right\}$, and that for each $x, y \in$ $X$, there is an operator $Q \in \mathscr{F}$ such that $\sum_{n=1}^{\infty} d\left(Q^{n} x, Q^{n} y\right)<\infty$. Then if $p=1$, either $S$ or $T$ has a fixed point. If $p>1$ then $S$ or $T$ has a periodic point, and if $p>1$ and $S$ and $T$ commute, either $S$ or $T$ has a fixed point.

Proof. Observe first that if $p=1$ (and so $\mathscr{F}$ consists of two operators), the conclusion is simply the preceding theorem with $N=2$. The hypothesis implies that, if $q=p$ !, then for every $x, y \in X$, either $\sum_{n=1}^{\infty} d\left(S^{n q} x, S^{n q} y\right)<\infty$ or $\sum_{n=1}^{\infty} d\left(T^{n q} x, T^{n q} y\right)<\infty$. The preceding remark enables us to conclude that either $S$ or $T$ has a periodic point (of order $q$ ). It only remains to show that either $S$ or $T$ has a fixed point if $S$ and $T$ commute.

We can assume without loss of generality that $T^{q} x=x$ for some $x \in X$. Then either $\sum_{n=1}^{\infty} d\left(T^{n q}(T x), T^{n q}(x)\right)<\infty$ or $\sum_{n=1}^{\infty} d\left(S^{n q}(T x), S^{n q}(x)\right)<\infty$. If the former, since $T^{q} x=x$, we see that $\sum_{n=1}^{\infty} d(T x, x)<\infty$, in which case we must have $d(T x, x)=0$ and so $T x=x$. We therefore assume the convergence of the latter series. We now either have

$$
\sum_{n=1}^{\infty} d\left(T^{n q}(T x), T^{n q}\left(S^{q} x\right)\right)<\infty \quad \text { or } \quad \sum_{n=1}^{\infty} d\left(S^{n q}(T x), S^{n q}\left(S^{q} x\right)\right)<\infty
$$

If the first of these inequalities holds, then since $T^{q} x=x$ and $S T=T S$, we have $\sum_{n=1}^{\infty} d\left(T^{n q}(T x), T^{n q}\left(S^{q} x\right)\right)=\sum_{n=1}^{\infty} d\left(T x, S^{q} x\right)<\infty$. So $T x=S^{q} x$. Substituting, we obtain $\sum_{n=1}^{\infty} d\left(S^{n q}\left(S^{q} x\right), S^{n q}(x)\right)<\infty$. But this is just $\sum_{n=1}^{\infty} d\left(T^{n+1} x, T^{n} x\right)$ $<\infty$, and so $T x=x$ as usual.

Finally, assume that both $\sum_{n=1}^{\infty} d\left(S^{n q}(T x), S^{n q}\left(S^{q} x\right)\right)<\infty$ and $\sum_{n=1}^{\infty} d\left(S^{n q}(T x)\right.$, $\left.S^{n q}(x)\right)<\infty$. Then $\sum_{n=1}^{\infty} d\left(S^{n q}(x), S^{n q}\left(S^{q} x\right)\right)<\infty$, which implies that $\left\{S^{n q} x\right\}_{n=1}^{\infty}$ is Cauchy. It therefore converges to a limit $u$, which satisfies $S^{q} u=u$. But then

$$
T^{q} u=T^{q}\left(\lim _{n \rightarrow \infty} S^{n q} x\right)=\lim _{n \rightarrow \infty}\left(T^{q} S^{n q} x\right)=\lim _{n \rightarrow \infty}\left(S^{n q} T^{q} x\right)=\lim _{n \rightarrow \infty} S^{n q} x=S u .
$$

So either $\sum_{n=1}^{\infty} d\left(T^{n q}(S u), T^{n q}(u)\right)<\infty$ or $\sum_{n=1}^{\infty} d\left(S^{n q}(S u), S^{n q}(u)\right)<\infty$. Either of these implies $\sum_{n=1}^{\infty} d(S u, u)<\infty$, and so $d(S u, u)=0$. Therefore $S u=u$.

We conclude with an example to demonstrate the necessity of assuming that the set of operators is finite.

EXAMPLE 2. We construct a complete metric space $(X, d)$ and a continuous map $T: X \mapsto X$ such that, for each $x, y \in X$, there is an integer $n=n(x, y)$ such that $\sum_{k=1}^{\infty} d\left(\left(T^{n}\right)^{k} x,\left(T^{n}\right)^{k} y\right)<\infty$, but $T$ has no fixed point. 
$X$ will be an increasing sequence $y_{1}<y_{2}<\cdots$ of real numbers, $d$ the absolute value metric, and $T$ the shift operator $T y_{k}=y_{k+1} . X$ will be the union of sets $A_{k}$, each of which is a finite subset of $[k, k+1) . X$ will therefore be a discrete metric space with no limit points, so $X$ will be complete, $T$ will be continuous and, as defined above, have no fixed points.

Let $A_{1}=\{1\}, A_{2}=\{2\} ;$ so $y_{1}=1, y_{2}=2$. The sets $A_{k}$ will be defined inductively to satisfy: $A_{k}=\left\{k=x_{1}^{(k)}<\cdots<x_{p_{k}}^{(k)}\right\}$;

for $k \geq 3, \quad$ if $\quad y_{j}=x_{1}^{(k)} \quad$ and $\quad y_{n}=x_{p_{k}}^{(k)}, \quad$ then $\quad y_{n}-y_{j}=\left(y_{j-1}-y_{j-2}\right) / 2$;

$$
\text { if } y_{j}, y_{j+1}, y_{j+2} \in A_{k} \text {, then } y_{j+2}-y_{j+1}=\left(y_{j+1}-y_{j}\right) / 2 \text {. }
$$

If $A_{1}, \ldots, A_{k-1}$ have been defined, (10) and (11) can be realized simply by specifying $p_{k}$. Let $p_{k}=\left(p_{1}+\cdots+p_{k-1}\right)$ !. Without loss of generality, assume $x=y_{n}, y=y_{p}$, and $n<p$. Assume that $y \in A_{k}$, and let $r=\left(p_{1}+\cdots+p_{k}+1\right)-n$. Note that $T^{r} x=x_{1}^{(k+1)}, T^{r} y=x_{1+p-n}^{(k+1)}$, and that

$$
\left(T^{r}\right)^{j} x=x_{1+(j-1) r}^{(k+1)}, \quad\left(T^{r}\right)^{j} y=x_{1+p-n+(j-1) r}^{(k+1)} .
$$

But since $r$ is a factor of $p_{k+1}$, for some value of $j$ we have

$$
\left(T^{r}\right)^{j} x=x_{1+(j-1) r}^{(k+1)}, \quad\left(T^{r}\right)^{j+1} x=x_{1}^{(k+2)},
$$

and since $p-n<r$, repeating this argument shows that, for any $j$, there is an integer $k$ such that both $\left(T^{r}\right)^{j} x$ and $\left(T^{r}\right)^{j} y$ belong to $A_{k}$. By construction,

$$
\sum_{j=1}^{\infty} d\left(T^{j r} x, T^{j r} y\right) \leq \sum_{j=1}^{\infty} 2^{-j} d(x, y)<\infty,
$$

establishing the example.

\section{References}

[1] F. E. Browder, 'On the convergence of successive approximations', Indag. Math. (N. S.) 30 (1968), 27-35.

[2] F. E. Browder and W. V. Petryshyn, 'The solution by iteration of nonlinear functional equations in Banach spaces', Bull. Amer. Math. Soc. (N. S.) 72 (1966), 571-576.

[3] J. Caristi, 'Fixed point theorems for mappings satisfying inwardness conditions', Trans. Amer. Math. Soc. 215 (1976).

[4] J. Dugundji, 'Positive definite functions and coincidences', Fund. Math. 90 (1976), 131-142.

[5] J. Dugundji and A. Granas, Fixed point theory. I (Polish Scient. Publ., Warszawa, 1982). 
[6] R. Engelking, General topology (Polish Scient. Publ., Warszawa, 1977).

[7] J. R. Jachymski, 'Equivalence of some contractivity properties over metrical structures', Proc. Amer. Math. Soc. 125 (1997), 2327-2335.

[8] J. R. Jachymski, B. Schröder and J. D. Stein, Jr., 'A connection between fixed-point theorems and tiling problems', J. Combin. Theory Ser. A (1999), to appear.

[9] J. D. Stein, Jr., 'A systematic generalization procedure for fixed-point theorems', Rocky Mountain J. Math. (1998), to appear.

[10] C. S. Wong, 'A fixed point theorem for a class of mappings', Math. Ann. 204 (1973), 97-103.

Institute of Mathematics

Technical University of Łódź

Żwirki 36

90-924 Łódź

Poland

e-mail: jachym@ck-sg.p.lodz.pl
Department of Mathematics

California State University

Long Beach

1250 Bellflower Blvd.

Long Beach, California 90840

USA

e-mail: jimstein@csulb.edu 